\title{
Correlation properties and time-resolved dynamics of linear polarizations emitted by single-mode vertical-cavity surface-emitting lasers subject to orthogonal optical injection
}

\author{
Ana Quirce, ${ }^{1,2}$ Pablo Pérez, ${ }^{1,2}$ Angel Valle, ${ }^{1, *}$ and Luis Pesquera ${ }^{1,2}$ \\ ${ }^{1}$ Instituto de Física de Cantabria, Consejo Superior de Investigaciones Cientificas-Universidad de Cantabria, \\ Avenida Los Castros s/n, E-39005 Santander, Spain \\ ${ }^{2}$ Departamento de Física Moderna, Universidad de Cantabria, Avenida Los Castros s/n, E-39005 Santander, Spain \\ ${ }^{*}$ Corresponding author: valle@ifca.unican.es
}

Received June 22, 2011; revised September 19, 2011; accepted September 19, 2011; posted September 21, 2011 (Doc. ID 149721); published October 26, 2011

\begin{abstract}
We present an experimental and theoretical study of the polarization and time-resolved nonlinear dynamics of a long-wavelength single-mode linearly polarized vertical-cavity surface-emitting laser (VCSEL) subject to orthogonal optical injection. Special attention is paid to the correlation properties of both linear polarizations emitted by the VCSEL. We measure simultaneous time traces of both linearly polarized output signals for several values of the bias current, the strength of the optical injection, and the frequency detuning between master and the free-running VCSEL. Different dynamical regimes, including periodic, period doubling, and irregular dynamics, are observed for both polarizations. For positive frequency detuning we usually find that the orthogonal polarization is the only polarization that contributes to the dynamics of the total power. Only for large values of the bias current and for small values of the optical injection strength, near the border of the periodic region, have we found correlated periodic dynamics in both linear polarizations. For negative frequency detuning we find anticorrelated dynamics in both linear polarizations. The average and dispersion of the time between consecutive pulses that appear in the anticorrelated regime increase with the injected power. The irregular dynamics, characterized by broadened power spectra, is related to large values of the dispersion of the time between pulses. A good overall qualitative agreement is found between our theoretical and experimental results. (C) 2011 Optical Society of America
\end{abstract} OCIS codes: $\quad 140.7260,140.1540,190.3100$.

\section{INTRODUCTION}

Optical injection in semiconductor lasers has been shown to lead to a wealth of complex dynamics and bifurcations, such as period-one state, period doubling, quasiperiodicity, chaos, and injection locking [1]. Nonlinear dynamics of optically injected semiconductor lasers is interesting from a practical point of view because the period-one oscillation state is one of the potential candidates for carrying message in a radioover-fiber (RoF) system [2]. Also optical injection can be used to reduce the laser linewidth, the mode partition noise, or for enhancing the modulation bandwidth without modifying the semiconductor laser design [3]. Interest in optical injection in vertical-cavity surface-emitting lasers (VCSELs) has recently increased $[\underline{4}, \underline{5}]$ due to the inherent advantages of this type of semiconductor laser. These include single-longitudinal mode operation, circular beam profile, reduced fabrication costs, ease of fabrication of two-dimensional arrays, etc. Studies of nonlinear dynamics of optically injected semiconductor lasers have been extended to VCSELs [ㅁ-15] since these devices offer additional degrees of freedom, like the presence of multiple transverse modes and the direction of the emitted polarization, when compared with their edge-emitting counterparts. The behavior of these systems not only includes the previously mentioned dynamical regimes but also polarization switching (PS) and optical bistability [ㅌ-20].
Early experiments considered short-wavelength devices in which the polarizations of both the VCSEL and the optical injection were parallel [6]. In this case optical injection produces similar effects to those found when light is injected in edge-emitting semiconductor lasers $[\underline{6}, \underline{13}]$. However, more attention has been paid to a configuration in which linearly polarized light from an external laser is injected orthogonally to the linear polarization of the free-running VCSEL, the so called orthogonal optical injection [16]. In contrast with edge emitters, VCSELs have an extra degree of freedom associated to the polarization of light. The specific injection scheme with orthogonally polarized master and slave lasers produces new features, like PS, that cannot be found when dealing with parallel optical injection. Nonlinear dynamics of VCSELs under orthogonal optical injection has been experimentally and theoretically analyzed for 850 [7-12] and $1550 \mathrm{~nm}$ [13-15] wavelength devices. Rich nonlinear dynamics, including period doubling, quasiperiodicity, injection locking, bistability, and chaos, have been found in short-wavelength VCSELs [7-12]. These devices were characterized by small values of birefringence and showed PS in absence of optical injection. Analysis of $1550 \mathrm{~nm}$ wavelength single transverse mode VCSELs subject to orthogonal optical injection have also shown limit cycle and period doubling dynamics, injection locking, irregular behavior [13-15], and bistability [18-20]. These long-wavelength devices were characterized by very large values of the birefringence parameter and by emission in a single linear 
polarization over the whole bias current range in absence of optical injection. Recent measurements of the polarizationresolved nonlinear dynamics have shown that the contribution of both linear polarizations to the dynamics of the total power depends on the sign of the frequency detuning between the injected light and the orthogonal linear polarization of the VCSEL [14]. These results were based on measurements of RF and optical spectra of both linear polarizations and of the total power. No analysis of the nonlinear dynamics was made in terms of simultaneous time traces of the power of both linear polarizations. Spectra were obtained from time averages of the signals [14], and hence relevant information contained in the temporal domain was missed.

In this work we extend the experimental study of [14] by measuring simultaneous time traces of both linearly polarized output signals of a long-wavelength VCSEL when subject to orthogonal optical injection. We have also measured time traces of the total power. Results are presented for different values of the bias current, the strength of the optical injection, and the frequency detuning. Different dynamical regimes including periodic, period doubling, and irregular dynamics are observed for both polarizations. An analysis is given of the correlation properties between both linear polarizations. We show that anticorrelation (correlation) is found for negative (positive) frequency detuning. The same qualitative results are obtained by using the well-established spin-flip model (SFM). We use this model in order to gain insight on the origin of the uncorrelated/correlated dynamics. We show that the average and dispersion of the time between consecutive pulses that appear in the anticorrelated regime increase with the injected power. We also show that the irregular dynamics is characterized by large values of the dispersion of the time between pulses that causes large broadening of power spectra. Good qualitative agreement is found between our experimental and theoretical results.

Our paper is organized as follows. The experimental setup is described in Section 2. Polarization-resolved time traces and their correlation properties are analyzed in Sections 3 and 4 , for small and large values of the bias current, respectively. In Section 4 we present our theoretical results. Finally, in Section 5, a summary and conclusions are presented.

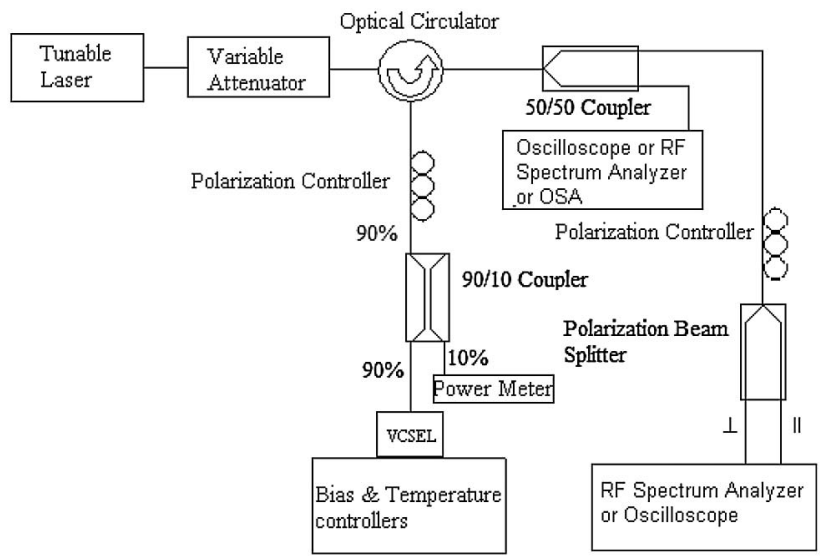

Fig. 1. Experimental setup of orthogonal optical injection in a VCSEL.

\section{EXPERIMENTAL SETUP}

The experimental all-fiber setup is presented in Fig. 1. The light from a tunable laser is injected into a quantum-well $1550 \mathrm{~nm}$ VCSEL (Raycan). Measurements are performed using the same VCSEL of $[13,14]$. The VCSEL bias current and temperature are controlled by a laser driver and a temperature controller, respectively. The temperature is held constant at $298 \mathrm{~K}$ during the experiments. A variable optical attenuator is included after the tunable laser to control the power of the optical injection. The output of the tunable laser is then injected into the VCSEL using a three-port optical circulator. Orthogonal optical injection is obtained by using a fiber polarization controller connected to the second port of the circulator. A 90/10 fiber directional coupler divides the optical path in two branches; the $10 \%$ branch is used to monitor the optical input power with a power meter, whereas the $90 \%$ output is directly connected to the VCSEL. The reflected output of the VCSEL is analyzed by connecting a 50/50 coupler to the third port of the circulator. One half of the power is used to obtain the time traces of the total power at the oscilloscope with bandwidth of $6 \mathrm{GHz}$. The oscilloscope is substituted by an electrical spectrum analyzer for measuring the RF spectrum of the total power or by an optical spectrum analyzer (OSA) for measuring the optical spectra. The other half of the power is directed to a polarization controller and a polarization beam splitter that selects the polarization direction in which the time traces or the RF spectra are measured. Simultaneous linearly polarized time traces are recorded by using two similar high-speed photodetectors (Thorlabs PDA8GS of $9 \mathrm{GHz}$ bandwidth) connected to the oscilloscope. The same detectors were connected before the $\mathrm{RF}$ analyzer for measuring the $\mathrm{RF}$ spectra of both linearly polarized signals.

The $L-I$ characteristics of the solitary VCSEL is shown in Fig. 2a of [14]. Fundamental transverse mode emission is obtained for all bias currents with a threshold current of $I_{\text {th }}=$ $1.64 \mathrm{~mA}$. The VCSEL emits in the same linear polarization (the "parallel" polarization) along the whole current range. The optical spectrum of the VCSEL biased well above threshold, $8 \mathrm{~mA}$, is illustrated in Fig. $2 \mathrm{~b}$ of [14]. Spectra with similar shapes are obtained for all bias currents. In that figure the two modes correspond to the two orthogonal polarizations of the fundamental transverse mode of the VCSEL. The main lasing polarization mode and the subsidiary mode that emits in the "orthogonal" polarization appear at wavelengths $\lambda_{\|}=$ $1536.6 \mathrm{~nm}$ and $\lambda_{\perp}=1537.09 \mathrm{~nm}$, respectively. The frequency splitting between the two orthogonal polarizations is $0.49 \mathrm{~nm}$ $(61.2 \mathrm{GHz})$, very large in comparison to those reported in short-wavelength devices [14].

\section{EXPERIMENTAL RESULTS: SMALL BIAS CURRENT}

We characterize the optical injection by its strength, given by the value of the optical power arriving at the VCSEL, $P_{\text {inj }}$, and by its frequency, $\nu_{\text {inj. }}$. We consider values of $\nu_{\text {inj }}$ that are close to the frequency of the perpendicular polarization, $\nu_{\perp}$, the frequency detuning being $\Delta \nu=\nu_{\text {inj }}-\nu_{\perp}$. The dynamical behavior of our system was summarized in [14] by using stability maps. In these maps different dynamical regimes, characterized by the RF spectra of the total power, were plotted in the $P_{\text {inj }}-\Delta \nu$ plane. The maps were obtained by fixing a value of $\Delta \nu$ and increasing $P_{\text {inj }}$ from low values. Figure 2 shows two 

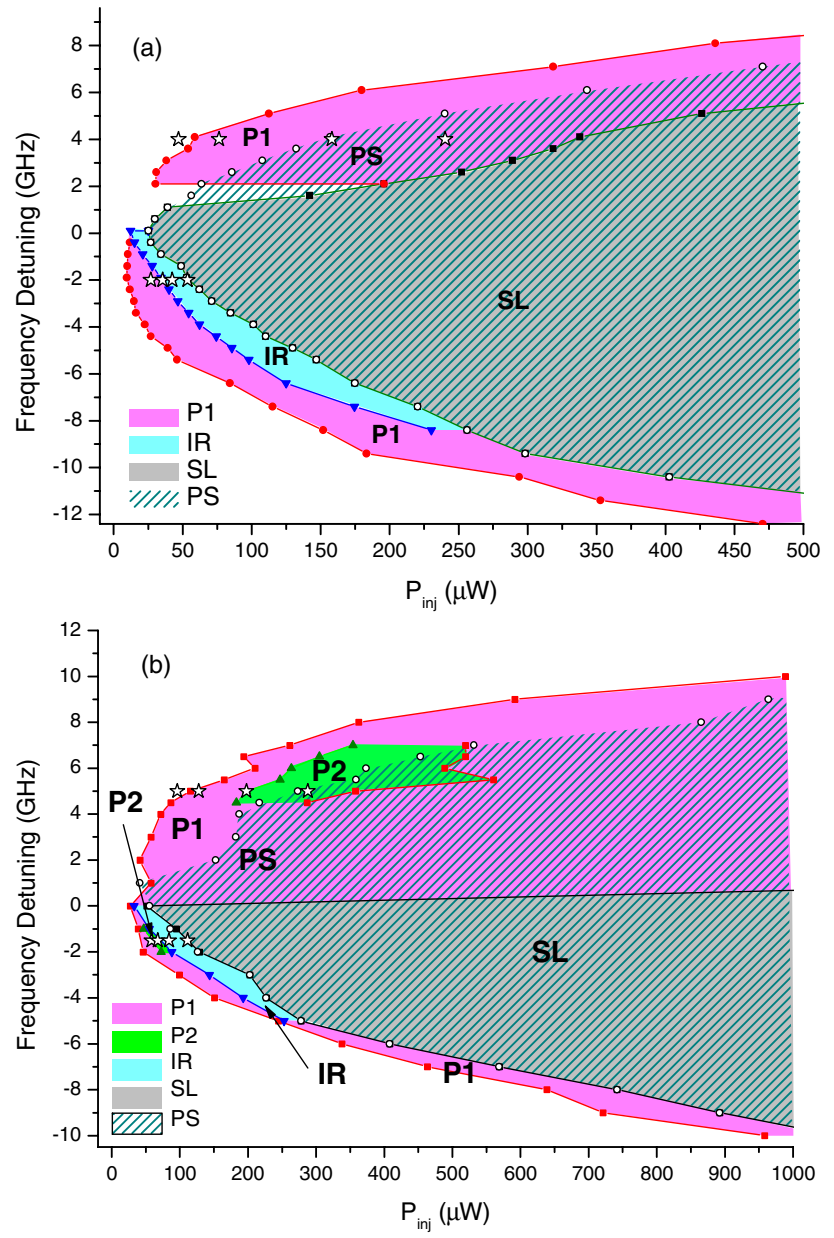

Fig. 2. (Color online) Stability map of the VCSEL subject to orthogonal optical injection for applied bias current of (a) $4 \mathrm{~mA}$ and (b) $8 \mathrm{~mA}$ [14]. Different regions are observed: SL, stable injection locking; P1, period 1; P2, period 2; IR, irregular dynamics; PS, polarization switching. The stars mark the situations analyzed in Figs. $3-\underline{6}$.

stability maps, obtained for bias currents of $I_{b}=4$ and $8 \mathrm{~mA}$ but plotted in a linear horizontal scale instead of a logarithmic one [14]. In Fig. 2 the region P1 shows periodic (period 1) behavior, P2 represents period doubling dynamics, region IR corresponds to irregular dynamics, and region SL represents the stable locking range. The injected power required for PS to the orthogonal polarization of the VCSEL is also shown in Fig. 2. When measuring these maps we considered P1 dynamics when the peak in the RF spectrum of the total power measured in the $\mathrm{RF}$ analyzer was $10 \mathrm{~dB}$ above the noise floor. A complete discussion of these diagrams was already given in [14]. We have chosen to include them in this work for the sake of clarity.

We now present in Figs. 3-6 the new results relative to the simultaneous measurement of linearly polarized temporal traces. We have chosen similar parameter values, $I_{b}, P_{\text {inj }}$, and $\Delta \nu$, to those of [14] in order to use the optical spectra shown in [14] in our discussion. RF spectra for the two polarizations and for the total power are shown in the left column of Fig. 3 for a fixed frequency detuning of $4 \mathrm{GHz}$, bias current of $4 \mathrm{~mA}\left(2.44 I_{\mathrm{th}}\right)$, and increasing values of $P_{\mathrm{inj}}$. The RF spectra shown in this work have been obtained by subtracting the RF spectra in the absence of light to the RF spectra with optical injection. We have done it in order to subtract the noise floor in the photodetector and RF analyzer. The corresponding simultaneous time traces of the power of both linear polarizations are shown in the right column of Fig. 3. In all the time traces presented in this work, we also include the sum of both powers (total power). Direct measurements of time traces of the total power show a similar behavior to that shown in the figures. The cases considered in Fig. $\underline{3}$ are identified in Fig. 2(a) with the upper row of white stars. We first analyze the transition to P1 dynamics that appears at low values of $P_{\text {inj. }}$ Figure 3(a) shows that a peak in the RF spectrum of the total power begins to appear near the frequency detuning. A similar peak appears in the RF spectrum of the orthogonal polarization power, while the spectrum corresponding to the parallel polarization is much flatter. Figure $\underline{3(\mathrm{~b})}$ shows that oscillations of small amplitude around the steady state characterize both linear polarizations and the total power. A clear P1 dynamics in the total and orthogonal polarized powers is obtained when increasing $P_{\text {inj }}$ as is shown in Figs. 3(c) and $3(\mathrm{~d})$. The power of the parallel polarization is still appreciable; however, the amplitude of their oscillations is very small without contributing to the periodic dynamics of the total power. This is also seen in Fig. 3(c) in which its RF spectrum is weak and flat. This periodic dynamics is caused by beating between the optical injection and the orthogonal mode of the VCSEL [14]. Figure 3(e) shows that the peaks appearing at the RF spectrum have shifted to larger frequency values. This is due to the "frequency pushing" effect observed in the optical spectra [14] that is caused by the effect of the increase of $P_{\text {inj }}$ on the refractive index. Figure 3(f) shows that the frequencies of the times traces of the total and orthogonal polarized powers have increased to the values at which RF spectra have their peaks. Further increase of $P_{\text {inj }}$ produces a decrease of the amplitude of the oscillations of the time traces [see Fig. 3(h)]. This is also shown by the decrease of the peaks in the RF spectrum [see Fig. 3(g)]. Figures $\underline{3(\mathrm{~g})}$ and $3(\mathrm{~h})$ also illustrate the situation in which PS and P1 dynamics are obtained. The optical spectrum of the total power is qualitatively similar to that of Fig. 4h in [14] showing that PS has occurred. Operation in the SL regime is illustrated in Figs. 3(i) and 3(j). The RF spectra and time traces of the total power and of the power of both linear polarizations become flat. The amplitude of the oscillations in the time traces of the total and orthogonal polarized powers are very small. We find an optical spectrum of the total power similar to that of Fig. $4 \mathrm{j}$ in [14] showing that the orthogonal polarization mode of the VCSEL is stably locked to the optical injection.

The correlation properties between the power of the parallel, $P_{\|}(t)$, and the orthogonal, $P_{\perp}(t)$, linear polarizations can be discussed in terms of their cross-correlation function, defined as

$$
C(\tau)=\frac{\overline{\left(P_{\|}(t+\tau)-\bar{P}_{\|}\right)\left(P_{\perp}(t)-\bar{P}_{\perp}\right)}}{\sigma_{\|} \sigma_{\perp}},
$$

where the bar means time averaging operation and $\sigma_{i}^{2}=\overline{\left(P_{i}(t)-\bar{P}_{i}\right)^{2}}, i=\|, \perp$. Temporal averages have been performed by using time series of $500 \mathrm{~ns}$ duration. The correlation between the power of the parallel and orthogonal linear polarizations is very weak for all the cases analyzed in Fig. 3. The absolute value of the cross-correlation functions obtained 

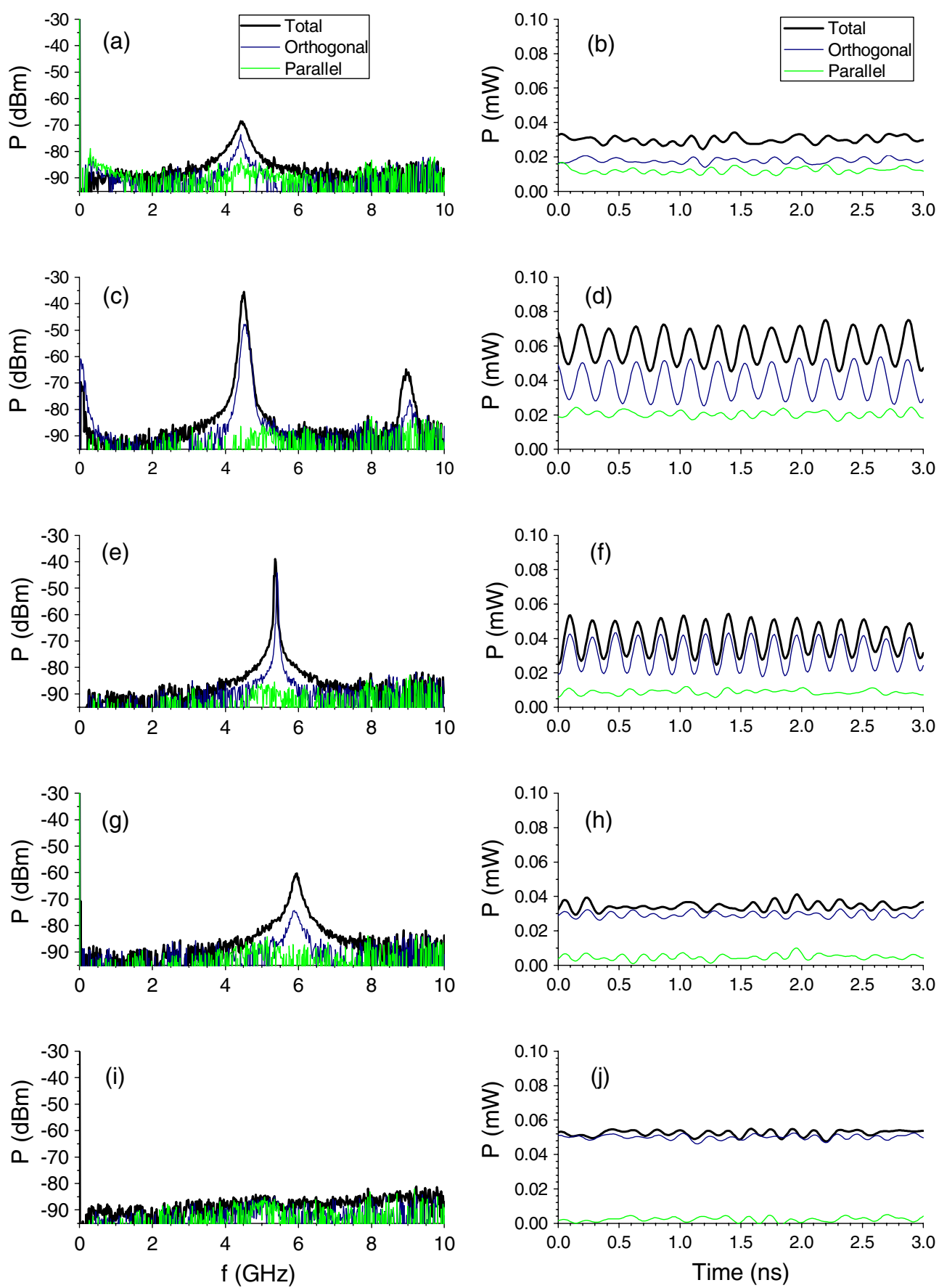

Fig. 3. (Color online) (Left) RF spectra of the total and polarized powers. (Right) Time traces of the total power and of the power of both linear polarizations. Several values of injected power are considered: (a), (b) $P_{\text {inj }}=47 \mu \mathrm{W}$, (c), (d) $P_{\text {inj }}=76.4 \mu \mathrm{W}$, (e), (f) $P_{\text {inj }}=158 \mu \mathrm{W},($ g), (h) $P_{\text {inj }}=240.3 \mu \mathrm{W}$, and (i), (j) $P_{\text {inj }}=1275.5 \mu \mathrm{W}$. The frequency detuning is $\Delta \nu=4 \mathrm{GHz}$, and the bias current is $4 \mathrm{~mA}$.

for the different cases considered in Fig. 3 is always below 0.08 .

We now analyze in Fig. 4 the case of a negative frequency detuning, $\Delta \nu=-2 \mathrm{GHz}$, with the same value of the applied bias current $(4 \mathrm{~mA})$. The cases analyzed in Fig. $\underline{4}$ are marked in Fig. 2(a) with the lower row of white stars. Figures $4(\mathrm{a})$ and $4(\mathrm{~b})$ show the P1 dynamics obtained for

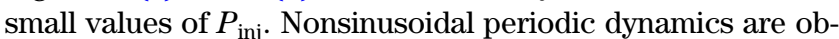
tained for both polarization modes, in contrast with the positive $\Delta \nu$ case. RF spectra of both polarizations and total power have peaks at a fundamental frequency $(1.9 \mathrm{GHz})$ and their harmonics that corresponds to the frequency detuning observed in the optical spectrum [14]. The large amplitude of the harmonic peaks in the RF spectrum is related to the nonsinusoidal shape of the temporal traces, in contrast to those obtained for positive $\Delta \nu$ [see Figs. $\underline{3(\mathrm{~d})}$ and $\underline{3(\mathrm{f})}$ ]. Figure 4(b) shows that the power of the parallel polarization consists of a train of large amplitude pulses that are responsible for the large amplitude pulses of the total power. The shoulder that appears in the total power after those pulses is mainly due to the contribution of the orthogonally polarized mode. Figure 4(b) shows that, after the decrease of $P_{\perp}$, a pulse of $P_{\|}$is fired with a certain delay. That delay is small enough for the pulse of $P_{\|}$to still coincide with the valley 
of the orthogonal power. Also the maximum of $P_{\perp}$ coincides with the absence of a pulse of $P_{\|}$. In this way the orthogonal and parallel polarized powers are anticorrelated. Figure $\underline{4(c)}$ shows that, for increasing $P_{\text {inj }}$ values, peaks of the RF spectra of both polarizations and total power appear at a smaller value of the frequency. This is a "frequency pulling" effect due to the change of refractive index induced by the increase of $P_{\text {inj }}[\underline{14}]$. The qualitative behavior of $P_{\|}$and $P_{\perp}$ shown in Fig. $\underline{4(\mathrm{~d})}$ is similar to that described in Fig. 4(b), although now the contributions of the pulses of $P_{\|}$and $P_{\perp}$ to the amplitude peaks and shoulders of the total power, respectively, are better defined. Comparison between Figs. 4(b) and 4(d) shows that the time between consecutive large amplitude peaks increases as $P_{\text {inj }}$ is increased. That time is usually called the interpulse time, $T$. The average of the interpulse time corresponding to the total power, $\langle T\rangle$, is 0.52 and $0.66 \mathrm{~ns}$ for Figs. 4(b) and $4(\mathrm{~d})$, respectively. The fundamental frequency in the RF spectra in Figs. 4(a) and 4(c) corresponds approximately to the inverse of $\langle T\rangle$.

Figure 4(f) illustrates the polarization-resolved time traces obtained for the irregular dynamics shown in Fig. 2(a). This dynamics is characterized by broad $\mathrm{RF}$ spectra in which the peaks tend to disappear as can be seen in Fig. 4(e). A better defined peak appears near $1 \mathrm{GHz}$ frequency that approximately corresponds to the inverse of $\langle T\rangle$ in Fig. $4(\mathrm{f})(\langle T\rangle=$ $0.93 \mathrm{~ns})$. Broadening of the RF spectra as $P_{\text {inj }}$ increases is related to a large increase of the interpulse time dispersion, $\sigma_{T}$. In fact, $\sigma_{T}$ is 12,40 , and $96 \mathrm{ps}$ for Figs. $\underline{4(\mathrm{~b})}$, $\underline{4(\mathrm{f})}$, and $\underline{4(\mathrm{~h})}$, respectively. The shoulder that appears after the large pulse of the total power changes its shape toward a plateau with small oscillations as $P_{\text {inj }}$ increases [see Figs. $\underline{4(\mathrm{f})}$ and $\underline{4(\mathrm{~h})}$ ]. The main contribution to that plateau is given by the orthogonal polarization. This means that, as the PS region is approached, the VCSEL is orthogonally polarized with a constant power during a longer time. In fact PS is obtained, simultaneously to SL, by increasing slightly $P_{\text {inj }}$ to $65 \mu \mathrm{W}$.

Anticorrelation between both linear polarizations is clear from the time traces of Fig. 4. This anticorrelation is confirmed by using the cross-correlation coefficient at $\tau=0$, $C(0)$ that takes the values $-0.54,-0.51,-0.48,-0.50$ for Figs. $\underline{4(\mathrm{~b})}, \underline{4(\mathrm{~d})}, \underline{4(\mathrm{f})}$, and $\underline{4(\mathrm{~h})}$, respectively. Information about the relative phase between the time traces of both polarizations can be obtained from the time at which $C(\tau)$ takes its minimum value. $C(\tau)$ have minimum values of -0.85 ,
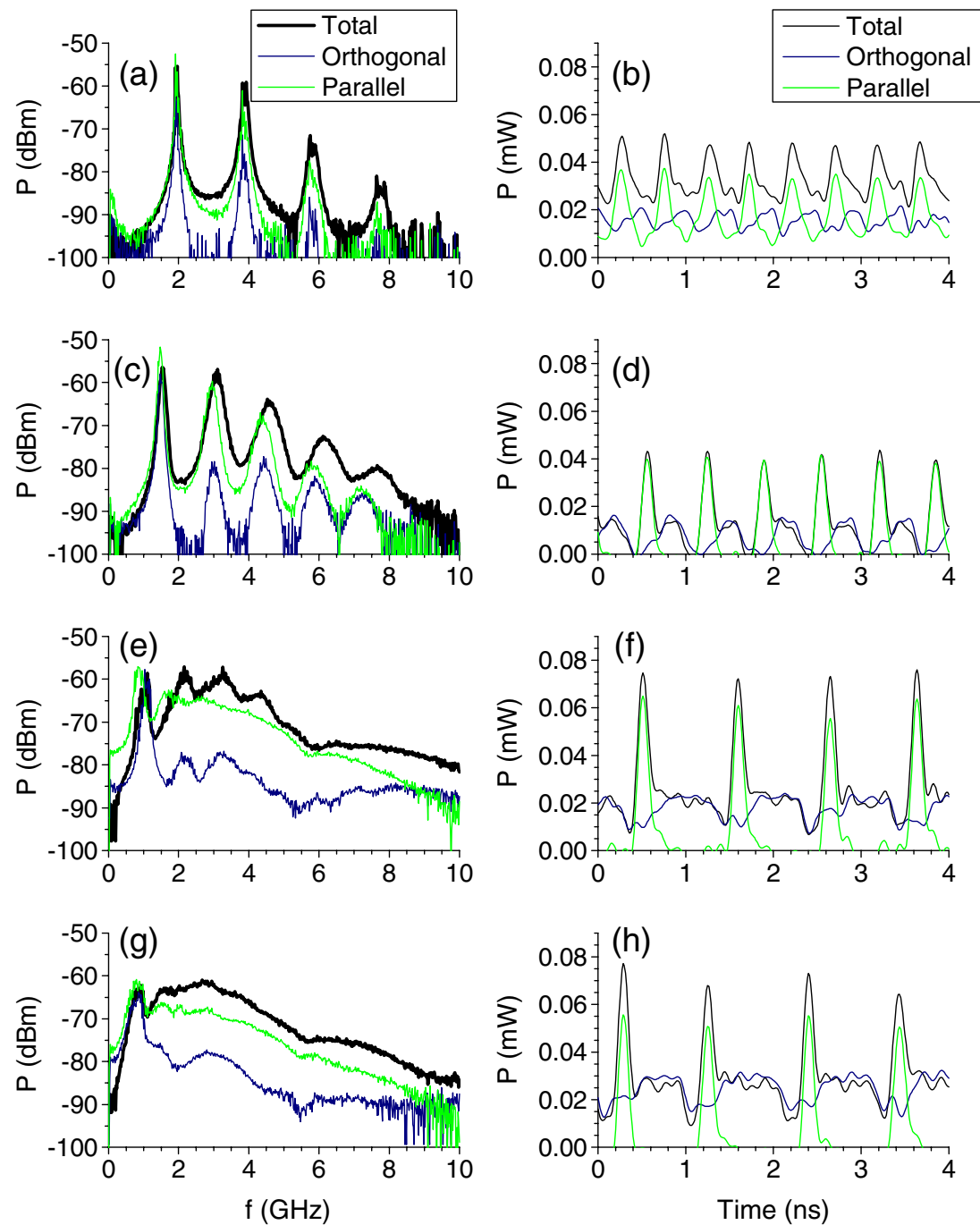

Fig. 4. (Color online) (Left) RF spectra of the total and polarized powers. (Right) Time traces of the total power and of the power of both linear polarizations. Several values of injected power are considered: (a), (b) $P_{\mathrm{inj}}=26.9 \mu \mathrm{W}$, (c), (d) $P_{\mathrm{inj}}=35.5 \mu \mathrm{W}$, (e), (f) $P_{\mathrm{inj}}=42.4 \mu \mathrm{W}$, and (g), (h) $P_{\text {inj }}=53.6 \mu \mathrm{W}$. The frequency detuning is $\Delta \nu=-2 \mathrm{GHz}$, and the applied bias current is $4 \mathrm{~mA}$. 
$-0.76,-0.64,-0.66$, at $\tau_{\min }=0.075,0.1,0.175,0.187 \mathrm{~ns}$ for Figs. 4(b), 4(d), 4(f), and $4(\mathrm{~h})$, respectively. $\tau_{\min }$ increases when increasing $\overline{P_{\text {inj }}}$ in such a way that the relative phase $\left(1-2 \tau_{\min } /\langle T\rangle\right) \pi$ is approximately constant [0.71 $\pi, 0.69 \pi$, $0.62 \pi, 0.67 \pi \mathrm{rads}$ for Figs. $\underline{4(\mathrm{~b})}, 4(\mathrm{~d}), \underline{4(\mathrm{f})}$, and $4(\mathrm{~h})$, respectively]. This relative phase is not exactly $\pi$ (antiphase) due to the delay of the emission of the parallel polarization pulse with respect to the decrease of the orthogonal one. Antiphase dynamics has been observed experimentally in multimode edge-emitting lasers with optical injection but at positive values of the frequency detuning [21]. Theoretical calculations in
VCSELs subject to orthogonal optical injection predicted that the two linearly polarized modes are anticorrelated at the time scale of slow oscillations while they exhibit an in-phase dynamics at the fast time scale of the relaxation oscillations [22]. Very recently, excitability and self-pulsating dynamics has been analyzed in optically injected quantum-dot and quantum-well laser diodes [23,24]. Time traces of the total power shown in Figs. 4(f) and 4(h) resemble the traces showing single-pulse excitability or self-pulsating behavior observed in $[\underline{23}, \underline{24}]$.
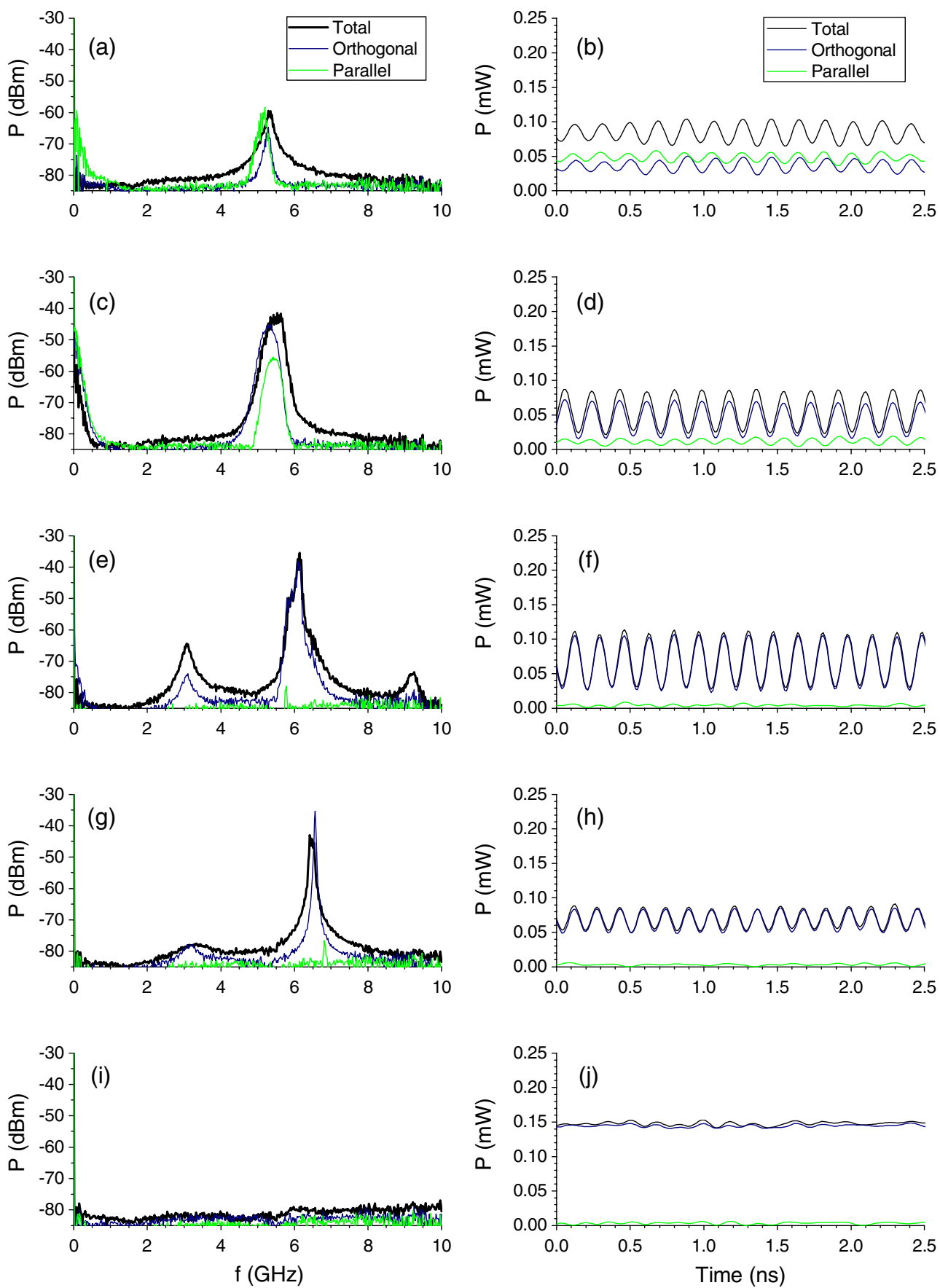

Fig. 5. (Color online) (Left) RF spectra of the total and polarized powers. (Right) Time traces of the total power and of the power of both linear polarizations. Several values of injected power are considered: (a), (b) $P_{\text {inj }}=96.1 \mu \mathrm{W}$, (c), (d) $P_{\text {inj }}=127.6 \mu \mathrm{W},(\mathrm{e}),(\mathrm{f}) P_{\text {inj }}=197.3 \mu \mathrm{W},(\mathrm{g})$, (h) $P_{\text {inj }}=287.8 \mu \mathrm{W}$, and (i), (j) $P_{\text {inj }}=3301.8 \mu \mathrm{W}$. The frequency detuning is $\Delta \nu=5 \mathrm{GHz}$, and the applied bias current is $8 \mathrm{~mA}$. 


\section{EXPERIMENTAL RESULTS: LARGE BIAS CURRENT}

We now discuss the dynamics of the system when the bias current is increased to $8 \mathrm{~mA}\left(4.9 I_{\mathrm{th}}\right)$. $\mathrm{RF}$ spectra, and time traces are shown in Fig. $\underline{5}$ for a fixed frequency detuning of $5 \mathrm{GHz}$ and increasing values of $P_{\text {inj. }}$. The cases considered in Fig. 5 are identified in Fig. 2(b) with the upper row of white stars. Figures $5(\mathrm{a})$ and $5(\mathrm{~b})$ illustrate the dynamics obtained for low values of $P_{\text {inj }}$ near the border of the P1 region. A peak in the RF spectra of the total power and of both linear polarizations appear near $5.5 \mathrm{GHz}$ that is slightly above the frequency detuning value. Figure $5(\mathrm{~b})$ shows that powers of both linear polarizations have a nearly sinusoidal oscillation. Both powers oscillate in a correlated way in such a way that both contribute to the sinusoidal time trace of the total power. A similar situation, with oscillations of larger amplitude, is obtained near the border but inside the $\mathrm{P} 1$ region as illustrated in Figs. 5(c) and 5(d).

Positive correlation between linear polarizations is also confirmed by using $C(0)$ that takes the values 0.75 and 0.65 for Figs. $\underline{5(b)}$ and $\underline{5(\mathrm{~d})}$, respectively. Information about the relative phase between the time traces of both polarizations can be obtained from the time at which $C(\tau)$ takes its maximum value. These times are $\tau_{\max }=-0.012,0$ ns, for Figs. $5(\mathrm{~b})$ and $\underline{5(\mathrm{~d})}$, respectively. The relative phase is given by $0.13 \pi$, 0 rads for Figs. $\underline{5(b)}$ and $\underline{5(\mathrm{~d})}$, respectively. This corresponds to a slight delay of the appearance of the maximum value of the orthogonal polarization with respect to the parallel one for Fig. 5(b). Only in the region near the border of P1 have we been able to observe correlated periodic dynamics in both linear polarizations. As we move further inside the $\mathrm{P} 1$ region, the RF peak corresponding to the parallel polarization disappears, while those corresponding to the orthogonal polarization and total power are maintained [14]. This situation, in which only the orthogonal polarization contributes to the dynamics of the total power, is found for all the values of $P_{\text {inj }}$ before SL is reached. Figures $\underline{5(\mathrm{e})}-\underline{5(\mathrm{~h})}$ are two examples that illustrate this behavior. In the situation illustrated in Figs. $\underline{5(\mathrm{e})}$ and 5(f), the power of the parallel polarization is still not negligible since the polarization of the VCSEL has not switched yet, as can be seen in Fig. 2(b). A weak positive correlation is found in Fig. 5(f): $C(\tau)$ has a maximum value of 0.24 at $\tau_{\max }=0 \mathrm{~ns}$. In-phase behavior of both polarizations is observed. In Figs. 5(g) and 5(h), in which PS has already occurred, no correlation is observed [the maximum value of $C(\tau)$ is 0.05 ]. Figures 5(e) and 5(g) show that the orthogonal polarization and the total power have period doubling dynamics. PS is observed in a periodic dynamical regime including period doubling behavior. Figures 5(i) and 5(j) show that SL dynamics is finally observed when $P_{\text {inj }}$ is increased to $3.3 \mathrm{~mW}$.

We now analyze the dynamics obtained for the case of a negative $\Delta \nu$ value. Figure $\underline{6}$ shows RF spectra and time traces for $\Delta \nu=-1.5 \mathrm{GHz}$. The cases considered in Fig. $\underline{6}$ are identified in Fig. 2(b) with the lower row of white stars. Figures $\underline{6(a)}$ and $6(\mathrm{~b})$ illustrate the P1 dynamics obtained at low values of $P_{\text {inj. }}$. The qualitative behavior of both polarizations is similar to that shown in Figs. 4(a) and 4(b): maxima (shoulders) of the total power are due to the large amplitude peaks (maxima) of the parallel (orthogonal) polarization. However, the approach to the SL dynamics as $P_{\text {inj }}$ is increased is different to that discussed in Section 3. Figure $\underline{6(\mathrm{c})}$ shows that P2 dynamics is obtained for both linear polarizations and for the total power when increasing $P_{\text {inj. }}$. In fact, one of the main differences with respect to the small bias current case is the presence of $\mathrm{P} 2$ regions for positive and negative $\Delta \nu$ values (see Fig. 2). Further increase of $P_{\text {inj }}$ leads to irregular behavior in both linear polarizations and in the total power as can be seen in Figs. $\underline{6(\mathrm{e})}$ and $\underline{6(\mathrm{f})}$. Figure $\underline{6(\mathrm{f})}$ shows that, before the SL region, time traces are much more irregular than those observed for smaller bias currents [see Fig. 4(h)]. Two large amplitude consecutive pulses are occasionally obtained in the total power [see Fig. 6(f) between 1 and 2 ns, between 2 and $3 \mathrm{~ns}$, and after $4 \mathrm{~ns}]$. The first (second) large amplitude pulse is due to a pulse of the orthogonal (parallel) polarized mode. Other parts of the time series resemble the results shown in Fig. 4(h): for instance, sometimes a single pulse is fired in the total power. This pulse is due to a large pulse of parallel polarized light [see Fig. 6 (f) between 0 and 1 ns and between 3 and $4 \mathrm{~ns}$. The more irregular behavior obtained for large values of the bias current is also observed in RF spectra: peaks of Fig. $6(\mathrm{e})$ are more smeared than those of Fig. $4(\mathrm{~g})$, especially those corresponding to the RF spectrum of the orthogonal polarization. The value of the interpulse time dispersion increases as $P_{\mathrm{inj}}$ is increased $\left[\sigma_{T}=7,10,200 \mathrm{ps}\right.$ for Figs. 6(b), 6(d), and 6(f), respectively]. The large value of $\sigma_{T}$ in Fig. 6 (f) is caused by the simultaneous presence of single-pulse and two-pulse packages. Finally, dynamics illustrating simultaneous achievement of SL and PS are also illustrated in Figs. $\underline{6(\mathrm{~g})}$ and $\underline{6(\mathrm{~h})}$. Some other similarities are observed with respect to the case of small bias current. First, the irregular behavior is also obtained only for negative values of $\Delta \nu$. Second, the orthogonal and parallel polarized powers are anticorrelated.

This anticorrelation is confirmed by using the cross-correlation coefficient at $\tau=0, C(0)$ that takes the values -0.37 , $-0.47,-0.67$ for Figs. $6(\mathrm{~b}), 6(\mathrm{~d})$, and $6(\mathrm{f})$, respectively. $C(\tau)$ have minimum values of $-0.94,-0.93,-0.77$ at $\tau_{\min }=0.062$, $0.062,0.075 \mathrm{~ns}$ for Figs. $6(\mathrm{~b}), 6(\mathrm{~d})$, and $6(\mathrm{f})$, respectively. $\tau_{\min }$ increases when increasing $P_{\text {inj }}$ in such a way that the relative phase $\left(1-2 \tau_{\min } /\langle T\rangle\right) \pi$ is approximately constant $[0.64 \pi$, $0.69 \pi$, and $0.74 \pi \mathrm{rads}$ for Figs. $\underline{6(\mathrm{~b})}, \underline{6(\mathrm{~d})}$, and $\underline{6(\mathrm{f})}$, respectively]. Both signals are not exactly in antiphase due to the delay of the emission of the parallel polarization pulse with respect to the decrease of the orthogonal one.

\section{THEORETICAL RESULTS}

In this section we are going to analyze from a theoretical point of view the correlation properties corresponding to the two linearly polarized modes emitted by the VCSEL subject to orthogonal optical injection. Our rate equation model for the polarization of a VCSEL is based on the SFM [25]. If the parameters are chosen such that the free-running VCSEL emits in the $y$ polarization, orthogonal optical injection is obtained by considering an external field along the $x$ direction. The SFM model equations are given by

$$
\begin{aligned}
\frac{d E_{x}}{d t}= & \kappa(1+i \alpha)\left(N E_{x}+i n E_{y}-E_{x}\right)-i \gamma_{p} E_{x}-\gamma_{a} E_{x} \\
& +\kappa E_{\mathrm{inj}} e^{i \Delta \omega t} \\
& +\sqrt{\beta_{s p} \gamma_{e} / 2}\left(\sqrt{N+n} \xi_{+}(t)+\sqrt{N-n} \xi_{-}(t)\right),
\end{aligned}
$$



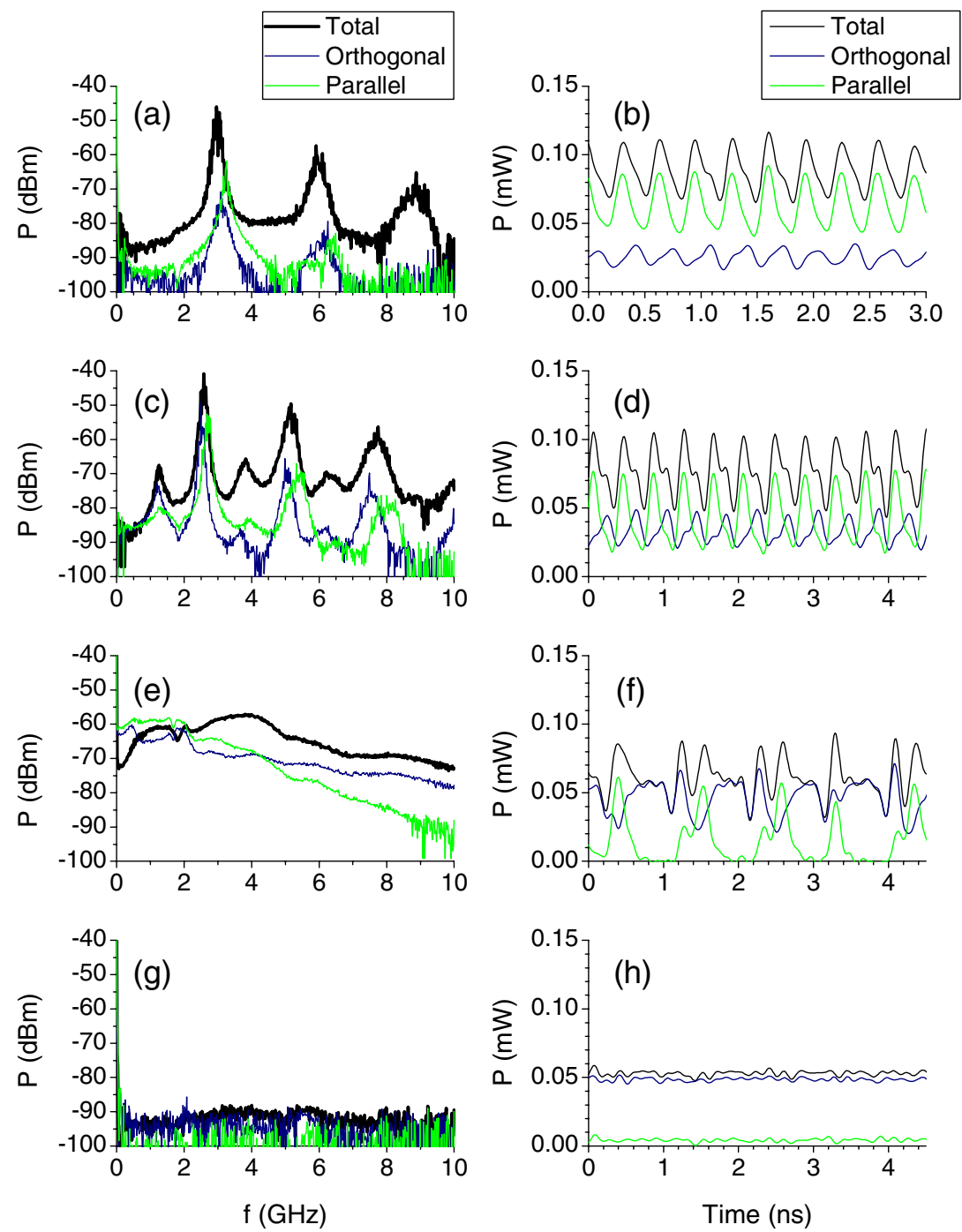

Fig. 6. (Color online) (Left) RF spectra of the total and polarized powers. (Right) Time traces of the total power and of the power of both linear polarizations. Several values of injected power are considered: (a), (b) $P_{\text {inj }}=58.5 \mu \mathrm{W}$, (c), (d) $P_{\text {inj }}=67.6 \mu \mathrm{W},(\mathrm{e}),(\mathrm{f}) P_{\text {inj }}=84 \mu \mathrm{W}$, and (g), (h) $P_{\text {inj }}=111.2 \mu \mathrm{W}$. The frequency detuning is $\Delta \nu=-1.5 \mathrm{GHz}$, and the applied bias current is $8 \mathrm{~mA}$.

$$
\begin{aligned}
\frac{d E_{y}}{d t}= & \kappa(1+i \alpha)\left(N E_{y}-i n E_{x}-E_{y}\right)+i \gamma_{p} E_{y}+\gamma_{a} E_{y} \\
& +\sqrt{\beta_{s p} \gamma_{e} / 2}\left(\sqrt{N+n} \xi_{+}(t)+\sqrt{N-n} \xi_{-}(t)\right)
\end{aligned}
$$

$$
\frac{d N}{d t}=-\gamma_{e}\left(N\left(1+\left|E_{x}\right|^{2}+\left|E_{y}\right|^{2}\right)\right)+\gamma_{e} \mu-i \gamma_{e} n\left(E_{y} E_{x}^{*}-E_{x} E_{y}^{*}\right),
$$

$$
\frac{d n}{d t}=-\gamma_{s} n-\gamma_{e} n\left(\left|E_{x}\right|^{2}+\left|E_{y}\right|^{2}\right)-i \gamma_{e} N\left(E_{y} E_{x}^{*}-E_{x} E_{y}^{*}\right),
$$

where $E_{x, y}$ are the two linearly polarized slowly varying components of the field and $N$ and $n$ are two carrier variables. $N$ accounts for the total population inversion between conduction and valence bands, while $n$ is the difference between the population inversions for the spin-up and spin-down radiation channels. The internal VCSEL parameters are as follows: $\kappa$ $\left(=125 \mathrm{~ns}^{-1}\right)$ is the field decay rate, $\gamma_{e}\left(=0.67 \mathrm{~ns}^{-1}\right)$ is the decay rate of $N, \gamma_{s}\left(=1000 \mathrm{~ns}^{-1}\right)$ is the spin-flip relaxation rate, $\alpha(=2.2)$ is the linewidth enhancement factor, $\mu$ is the normalized injection current, $\gamma_{a}\left(=2 \mathrm{~ns}^{-1}\right)$ is the linear dichroism, and $\gamma_{p}\left(=192 \mathrm{~ns}^{-1}\right)$ is the linear birefringence. The fluctuating nature of the spontaneous emission (with a fraction of spontaneous emission photons that goes into the laser mode of $\left.\beta_{s p}=10^{-4}\right)$ is included in our calculations since $\xi_{+}(t)$ and $\xi_{-}(t)$ are complex Gaussian noise terms of zero mean and time correlation given by $\left\langle\xi_{i}(t) \xi_{j}^{*}\left(t^{\prime}\right)\right\rangle=2 \delta_{i j} \delta\left(t-t^{\prime}\right)$. The optical injection parameters are $E_{\text {inj }}$ and $\Delta \omega . E_{\text {inj }}$ is the injected field amplitude and $\Delta \omega$ is defined as the difference between the angular frequency of the injected light, $\omega_{\mathrm{inj}}$, and a reference angular frequency intermediate between those of the $x$ and $y$ linear polarizations, i.e., $\Delta \omega=\omega_{\text {inj }}-\left(\omega_{x}+\omega_{y}\right) / 2$. We have integrated Eqs. (2)-(5) by using the same numerical method for stochastic differential equations used in [26] with an integration time step of $0.01 \mathrm{ps}$. The evolution of $E_{x}$ is such that the $k E_{\text {inj }} \exp (i \Delta \omega t)$ term appears in the equation for $d E_{x} / d t$. However, no injection term appears in the equation for $d E_{y} / d t$. In this way the increase of the optical injection strength (given by $k E_{\text {inj }}$ ) only affects directly to $E_{x}$, producing 
the PS to the $x$-polarized mode that has been usually observed in VCSELs since the pioneering work of Pan et al. [16].

We have chosen parameters based on those reported by Al-Seyab et al. [15] for a long-wavelength VCSEL similar to our device. With our parameters, the free-running VCSEL is emitting in the parallel polarized mode ( $y$ mode) for all the bias current values, as in the experimental situation. Also, the frequency of the parallel polarized mode is higher than that of the orthogonal linear polarization ( $x$ mode): $\nu_{y}>\nu_{x}$, like for the VCSEL used in our experiments. The main change with respect to the parameters of [15] is the value of the linear dichroism since we have checked that the value reported in [15] leads to light-current characteristics in which multiple $\overline{\mathrm{PSs}}$ occur as the bias current is increased. Multiple PS appear because both polarizations are stable with the parameters chosen in [15].

Our first theoretical results illustrate the case in which positive correlation is found between both linear polarizations. Figure 7 (a) shows time traces of the total power and of the power of both polarizations when the bias current is five times the threshold value $(\mu=5)$ and $\Delta \nu=\nu_{\text {inj }}-\nu_{x}=5 \mathrm{GHz}$ (similar to Fig. 5) and $E_{\mathrm{inj}}=0.29$. Qualitative behavior is similar to that shown in Fig. 5(b): both linearly polarized powers oscillate in a correlated way in such a way that both contribute to the almost sinusoidal time trace of the total power. Figure 7(b) shows the time evolution of the total population inversion. $N$ is characterized by a sinusoidal evolution of small amplitude. The decrease of $N$ is related to the increase of the power in both polarizations. The frequency of the oscillations is around $6 \mathrm{GHz}$, larger than the value of $\Delta \nu$. This is due to the frequency pushing effect that appears due to the positive value of $\alpha$ : when $\Delta \nu>0$ the resonance wavelength of the orthogonal

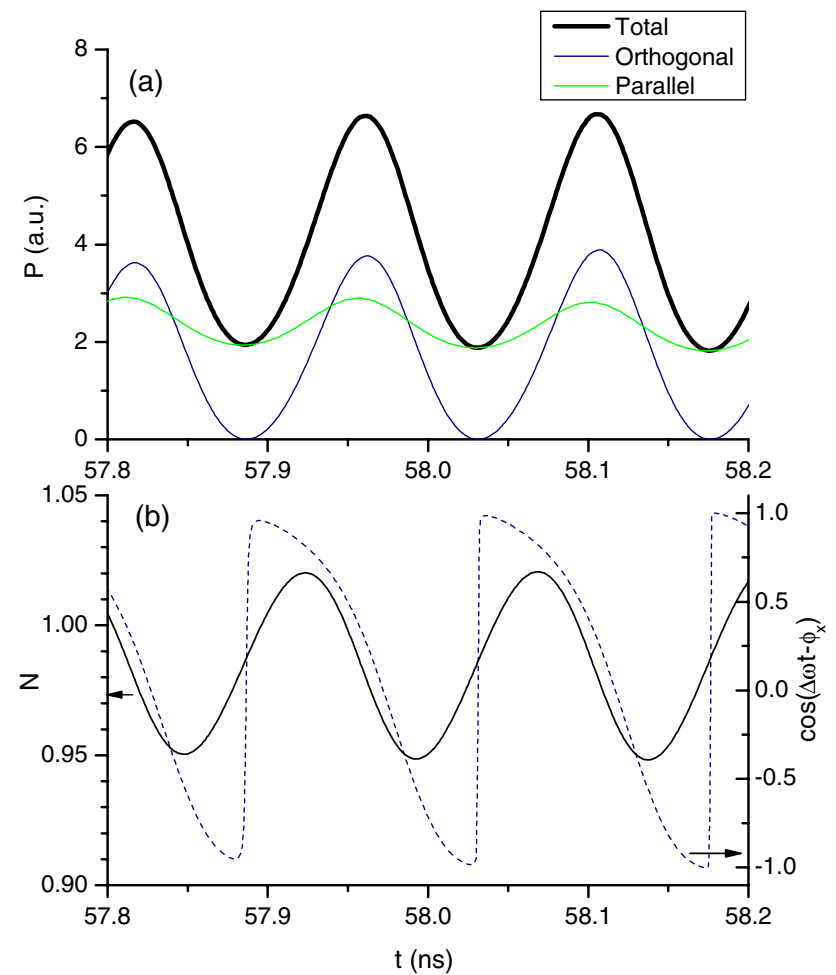

Fig. 7. (Color online) Theoretical time traces of the (a) total power and power of both linear polarizations and (b) total population inversion (solid line) and $k E_{\text {inj }} \cos \left(\Delta \omega t-\phi_{x}(t)\right)$ term (dashed line). The frequency detuning is $\Delta \nu=5 \mathrm{GHz}, \mu=5$, and $E_{\mathrm{inj}}=0.29$. mode moves away from the injected wavelength as $P_{\text {inj }}$ increases. This also means that optical injection is less effective in creating nonlinear effects. In fact the time evolution of the quantities shown in Fig. 7 is nearly sinusoidal. A positive correlation between linear polarizations of $C(0)=0.87$ is obtained. $C(\tau)$ takes its maximum value at $\tau_{\max }=0 \mathrm{~ns}$. The relative phase is given by $0.12 \pi$ rads. This corresponds to a slight delay of the appearance of the maximum value of the orthogonal polarization with respect to the parallel one, in agreement with our experimental results shown in Fig. 5(b).

Figure 8 shows the time evolution of the total power, the power of both polarizations and $N$, and the RF spectra when $\mu=2.44$ and $\Delta \nu=-2 \mathrm{GHz}$ (similar to Fig. 4) for two different values of the injected power, $E_{\mathrm{inj}}=0.0445$ and 0.047. Qualitative behavior is similar to that shown in Figs. 4(b) and 4(f), respectively. Anticorrelated periodic dynamics are obtained for both polarization modes. Increasing the value of $E_{\text {inj }}$ to 0.0495 produces simultaneous PS and SL, similar to our experimental results. Nonlinear effects are stronger than those obtained for $\Delta \nu>0$ because an increase of $P_{\text {inj }}$ makes the resonance wavelength of the orthogonal mode approach the injected wavelength. In fact, nonlinear oscillations of all the variables appear in Fig. $\underline{8}$, in contrast to the sinusoidal evolutions shown in Fig. 7 . The strength of nonlinear oscillations increase as $P_{\text {inj }}$ is increased [see for instance Figs. $\underline{8(\mathrm{c})}$ and $8(\mathrm{~d})]$. Figures $\underline{8(\mathrm{c})}$ and $\underline{8(\mathrm{~d})}$ also show that faster decrease of $N$ is related to the excitation of the parallel polarized pulse, while the low recovery of $N$ is accompanied by the emission of the wider orthogonally polarized pulse. The presence of these two different evolution stages of $N$ is related to the observed anticorrelated behavior between both polarizations. We have performed calculations of $C(\tau)$ by using theoretical time series of the total power of $819.2 \mathrm{~ns}$ duration. $C(0)$ takes the values -0.96 and -0.51 for Figs. $8(\mathrm{a})$ and $8(\mathrm{~b})$, respectively. $C(\tau)$ have minimum values of $\overline{-0.96},-0.71$ at $\tau_{\min }=$ $0.01,0.12 \mathrm{~ns}$ for Figs. $\underline{8(\mathrm{a})}$ and $\underline{8(\mathrm{~b})}$, respectively. $\tau_{\min }$ increases when increasing $P_{\text {inj }}$ in such a way that the relative phase $\left(1-2 \tau_{\min } /\langle T\rangle\right) \pi$ is approximately constant $[0.99 \pi$, $0.92 \pi$ rads for Figs. $8(\mathrm{a})$ and $8(\mathrm{~b})$, respectively], in qualitative agreement with the experimental results reported in Fig. 4.

The interpulse time increases as $P_{\text {inj }}$ is increased as can be seen in Figs. 8(a) and 8(b). Theoretical values of $\langle T\rangle$ are 2.4, $3.06 \mathrm{~ns}$ for Figs. 8(a) and 8(b), respectively. The interpulse time dispersion, $\overline{\sigma_{T}}$, also increases from 25 to $40.9 \mathrm{ps}$ when going from Fig. 8(a) to Fig. 8(b). The behavior of both $\langle T\rangle$ and $\sigma_{T}$ as a function of $P_{\text {inj }}$ is in qualitative agreement with the corresponding experimental results reported in Section 3. The lower row of Fig. 8 shows the RF spectrum of the total power and of the power of both linear polarizations for $E_{\text {inj }}=$ 0.0445 and 0.047 . A similar spectra broadening to that reported in Figs. $4(\mathrm{c})$ and $4(\mathrm{~g})$ is obtained when $E_{\text {inj }}$ is increased. This broadening is related to the observed increase of $\sigma_{T}$ : decreasing the strength of spontaneous emission noise leads to the decrease of $\sigma_{T}$ and narrower spectra. Comparison with experimental data also shows that simulations show stronger $\mathrm{RF}$ modes than in the experiment. There are several reasons that can contribute for this difference. First, experimental time traces (see Fig. 4) have more dispersion in the peaks height than that found theoretically (see Fig. 8). Second, although we have used angled polished ferrule connectors in our setup to minimize the optical feedback in the VCSEL, 

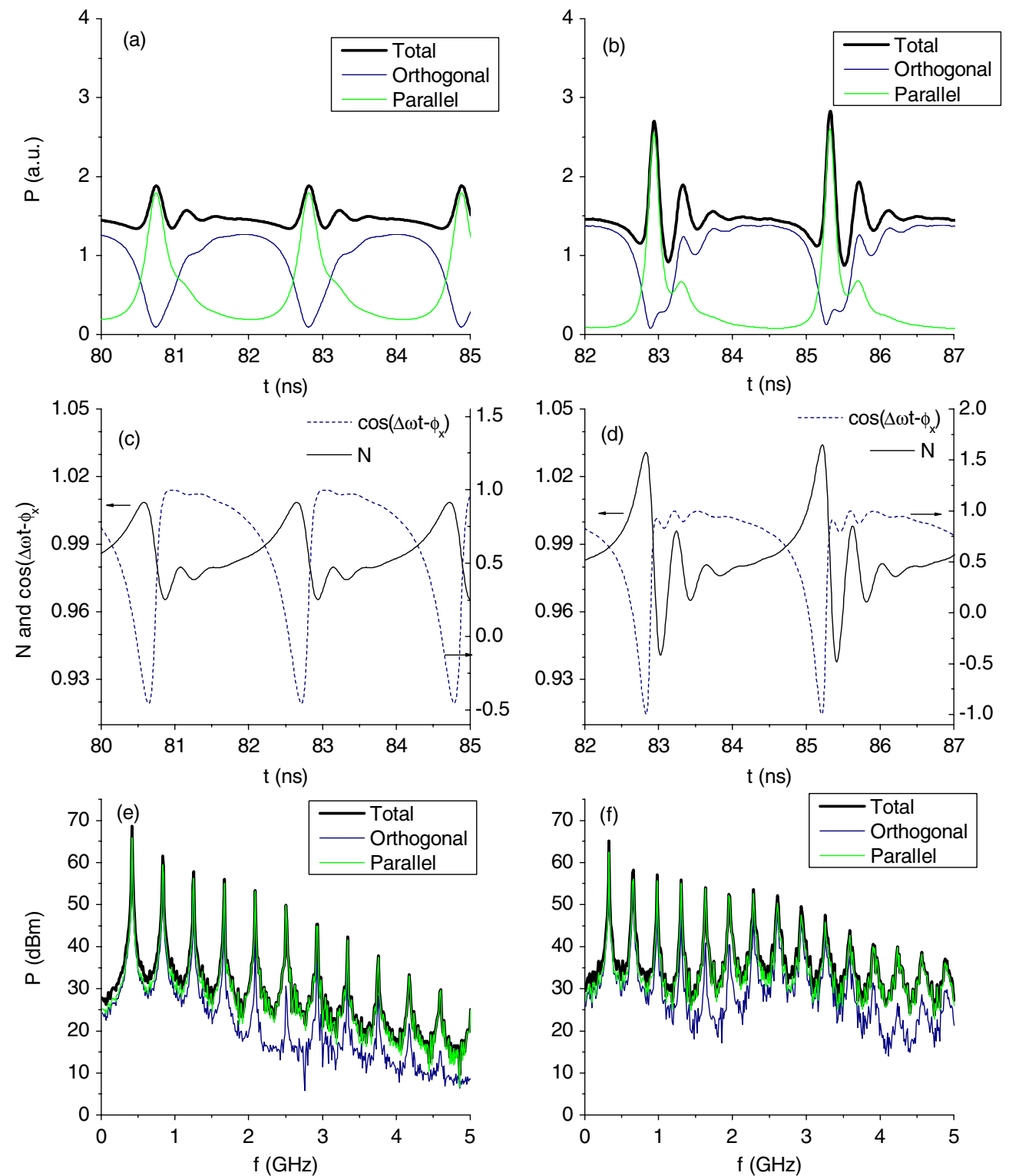

Fig. 8. (Color online) Theoretical time traces of the (a), (b) total power and power of both linear polarizations, (c), (d) total population inversion and $k E_{\text {inj }} \cos \left(\Delta \omega t-\phi_{x}(t)\right)$ term, and (e), (f) RF spectra. The frequency detuning is $\Delta \nu=-2 \mathrm{GHz}, \mu=2.44$, (left) $E_{\text {inj }}=0.0445$, and (right) $E_{\text {inj }}=0.047$.

this effect can be still present and degrade the signal to noise in our experimental RF spectra. Third, and more importantly, we do not know for sure the parameters of our VCSEL. In this way extraction of the laser parameters would be desirable for obtaining a better agreement with the experiments.

\section{DISCUSSION AND CONCLUSIONS}

For negative (positive) frequency detunings, we have obtained anticorrelated (correlated) operating regimes. We explain this behavior because there is a dynamics of competence between polarizations for $\Delta \nu<0$ while that dynamics disappears when $\Delta \nu>0$. For $\Delta \nu<0$, anticorrelated dynamics appears when the system is approaching the injection locking regime. In this approach [see Figs. 8(a) and 8(b)], the orthogonal polarization tries to reach the constant values of amplitude and phase that characterize the injection locked regime, but it is not able to do it because of the effect of the phase of the injected field. Figure $\underline{8}$ also shows that the average power of the orthogonal $(x)$ polarization is larger than that of the parallel $(y)$ polarization. As discussed in Section 3 , optical injection produces a frequency pulling effect such that the powers of both polarizations oscillate at a frequency that is smaller than the frequency detuning. That oscillation frequency coincides with the frequency detuning observed in the optical spectrum that we will call "effective frequency detuning." Optical phase of the orthogonal polarization is also oscillating at the effective frequency detuning. The orthogonal polarization cannot reach the stable locked behavior because the optical injection becomes out of phase with respect to that polarization.

In order to clarify this point, we have made further calculations analyzing the amplitude, $A_{i}(t)$, and the phase, $\phi_{i}(t)$, of the electrical fields of both linear polarizations, $E_{i}(t)=$ $A_{i}(t) \exp \left(i \phi_{i}(t)\right)$. When writing Eqs. (2)-(5) using the new variables, the evolution of the amplitude of the $x$ polarization is such that a $k E_{\text {inj }} \cos \left(\Delta \omega t-\phi_{x}(t)\right)$ term appears in the 
equation for $d A_{x} / d t$. We plot in Figs. $\underline{8(\mathrm{c})}$ and $\underline{8(\mathrm{~d})}$ that term together with $N$. When the optical injection becomes out of phase with respect to the $x$ polarization, the cosinus term decreases, causing the fall of $A_{x}$ [see Figs. 8(a) and 8(b)]. Simultaneously, $N$ increases until it decreases sharply with the fire of a large amplitude $y$ pulse. In this way one of the two polarization states exhibit large amplitude pulses, while the other exhibits the shoulders. This is the explanation of the anticorrelation observed between orthogonal and parallel polarizations, which is typical of a dynamics of competence between polarizations.

For positive frequency detunings, the system is far from the injection locking regime in such a way that the average powers of the both polarizations are similar [see Fig. 7(a)]. The optical phase of the $x$ polarization is oscillating at the effective frequency detuning that is larger than the frequency detuning due to the frequency pushing effect. Again, the optical injection can be out of phase with respect to the orthogonal polarization as is seen in Fig. $\underline{7(b)}$ in which the $k E_{\text {inj }} \cos \left(\Delta \omega t-\phi_{x}(t)\right)$ term has been plotted. The phase term induces a modulation of the amplitude of the $x$-polarization power. That modulation causes a small amplitude sinusoidal dependence of $N$, in which $A_{x}$ has the typical $\pi / 2$ delay with respect to $N$. The modulation of $A_{y}$ is only due to the modulation of $N$ because the optical injection has no effect on this polarization. The small signal change of $N$ causes a small amplitude modulation of $A_{y}$ that has again the $\pi / 2$ delay with respect to $N$. In this way correlation between orthogonal and parallel polarizations is obtained.

Summarizing, in this work we have made a theoretical and experimental study of the polarization-resolved nonlinear dynamics of a $1550 \mathrm{~nm}$ single-mode VCSEL subject to orthogonal optical injection. Simultaneous measurements of the time traces of the power of both linear polarizations have permitted us to evaluate their contribution to the dynamics of the total power. We have characterized the periodic, period doubling, and irregular dynamics that appear for both polarizations in terms of time traces and power spectra. We have obtained that the correlation properties of both linear polarizations depend on the frequency detuning between the injected frequency and the frequency of the suppressed orthogonal mode of the solitary laser. For positive frequency detuning values, we have usually found that orthogonal polarization is the only polarization that contributes to the dynamics of the total power. Only correlated periodic dynamics in both linear polarizations has been found near the border of the periodic region, when the bias current is large and optical injection strength is small. For negative frequency detuning values, the correlation properties change because we find anticorrelated dynamics in both linear polarizations. This dynamical regime is characterized by a larger influence of nonlinear effects that produce anticorrelated trains of pulses in both linear polarizations. These trains of pulses have been characterized by the average and dispersion of the time elapsed between consecutive pulses, the interpulse time. We have shown that both the average and dispersion of the interpulse time increase as the injected power is increased. We have also shown that the appearance of broad power spectra that characterizes the irregular dynamics is related to large values of the dispersion of the interpulse time. We have also analyzed the relative phase between the oscillations observed in the time traces of the optical power of both polarizations. Good qualitative agreement has been demonstrated between theory and experiment. Our results show that optically injected $1550 \mathrm{~nm}$ VCSELs are sources of stable periodic oscillations. Low-cost characteristics of these systems makes them potential candidates for generation of stable RF signals for use in RoF applications [27].

\section{ACKNOWLEDGMENTS}

The authors would like to acknowledge Dr. Antonio Hurtado and Prof. Michael Adams from the University of Essex (UK) for fruitful discussions. This work has been funded in part by the Ministerio de Ciencia e Innovación, Spain, under project TEC2009-14581-C02-02 and by European Commission project "Towards a PHOtonics liquid state machine based on delayCoUpled Systems" (PHOCUS) (FP7-ICT-2009-C-240763).

\section{REFERENCES}

1. S. Wieczorek, B. Krauskopf, T. B. Simpson, and D. Lenstra, "The dynamical complexity of optically injected semiconductor lasers," Phys. Rep. 416, 1-128 (2005).

2. S. C. Chan, S. K. Hwang, and J. M. Liu, "Radio-over-fiber AM-to-FM upconversion using an optically injected semiconductor laser," Opt. Lett. 31, 2254-2256 (2006).

3. G. H. M. van Tartwijk and D. Lenstra, "Semiconductor lasers with optical injection and feedback," Quantum Semiclass. Opt. 7, 87-143 (1995).

4. F. Koyama, "Recent advances of VCSEL photonics," J. Lightwave Technol. 24, 4502-4513 (2006).

5. D. Parekh, B. Zhang, X. Zhao, Y. Yue, W. Hofmann, M. C. Amann, A. Willner, and C. J. Chang-Hasnain, "Long distance single-mode fiber transmission of multimode VCSELs by injection locking," Opt. Express 18, 20552-20557 (2010).

6. H. Li, T. Lucas, J. G. McInerney, M. Wright, and R. A. Morgan, "Injection locking dynamics of vertical cavity semiconductor lasers under conventional and phase conjugate injection," IEEE J. Quantum Electron. 32, 227-235 (1996).

7. Y. Hong, P. S. Spencer, S. Bandyopadhyay, P. Rees, and K. A. Shore, "Polarization resolved chaos and instabilities in a VCSEL subject to optical injection," Opt. Commun. 216, 185-187 (2003).

8. J. Buesa, I. Gatare, K. Panajotov, H. Thienpont, and M. Sciamanna, "Mapping of the dynamics induced by orthogonal optical injection in vertical-cavity surface-emitting lasers," IEEE J. Quantum Electron. 42, 198-207 (2006).

9. I. Gatare, M. Sciamanna, M. Nizette, and K. Panajotov, "Bifurcation to polarization switching and locking in vertical-cavity surface-emitting lasers with optical injection," Phys. Rev. A 76, 031803(R) (2007).

10. K. Panajotov, I. Gatare, A. Valle, H. Thienpont, and M. Sciamanna, "Polarization- and transverse-mode dynamics in optically injected and gain-switched vertical-cavity surfaceemitting lasers," IEEE J. Quantum Electron. 45, 1473-1481 (2009).

11. M. Nizette, M. Sciamanna, I. Gatare, H. Thienpont, and K. Panajotov, "Dynamics of vertical-cavity surface-emitting lasers with optical injection: a two-mode model approach," J. Opt. Soc. Am. B 26, 1603-1613 (2009).

12. D. L. Boiko, G. M. Stéphan, and P. Besnard, "Fast polarization switching with memory effect in a vertical cavity surface emitting laser subject to modulated optical injection,” J. Appl. Phys. 86, 4096-4099 (1999).

13. A. Hurtado, A. Quirce, A. Valle, L. Pesquera, and M. J. Adams, "Nonlinear dynamics induced by parallel and orthogonal optical injection in $1550 \mathrm{~nm}$ vertical-cavity surface-emitting lasers (VCSELs)," Opt. Express 18, 9423-9428 (2010).

14. P. Pérez, A. Quirce, L. Pesquera, and A. Valle, "Polarizationresolved nonlinear dynamics induced by orthogonal optical injection in long-wavelength VCSELs," IEEE J. Sel. Top. Quantum Electron. 17, 1228-1235 (2011). 
15. R. Al-Seyab, K. Schires, N. A. Khan, A. Hurtado, I. D. Henning, and M. J. Adams, "Dynamics of polarized optical injection in $1550 \mathrm{~nm}$ VCSELs: theory and experiments," IEEE J. Sel. Top. Quantum Electron. 17, 1242-1249 (2011).

16. Z. G. Pan, S. Jiang, M. Dagenais, R. A. Morgan, K. Kojima, M. T. Asom, and R. E. Leibenguth, "Optical injection induced polarization bistability in vertical-cavity surface-emitting lasers," Appl. Phys. Lett. 63, 2999-3001 (1993).

17. K. H. Jeong, K. H. Kim, S. H. Lee, M. H. Lee, B. S. Yoo, and K. A. Shore, "Optical injection-induced polarization switching dynamics in $1.5 \mu \mathrm{m}$ wavelength single-mode vertical-cavity surface-emitting lasers," IEEE Photon. Technol. Lett. 20, 779-781 (2008).

18. A. Hurtado, I. D. Henning, and M. J. Adams, "Two-wavelength switching with a $1550 \mathrm{~nm}$ VCSEL under single orthogonal optical injection," IEEE J. Sel. Top. Quantum Electron. 14, 911-917 (2008).

19. A. Valle, M. Gomez-Molina, and L. Pesquera, "Polarization bistability in $1550 \mathrm{~nm}$ wavelength single-mode verticalcavity surface-emitting lasers subject to orthogonal optical injection," IEEE J. Sel. Top. Quantum Electron. 14, 895-902 (2008).

20. M. S. Torre, A. Quirce, A. Valle, and L. Pesquera, "Wavelengthinduced polarization bistability in $1550 \mathrm{~nm}$ VCSELs subject to orthogonal optical injection,” J. Opt. Soc. Am. B 27, 2542-2548 (2010).
21. S. Osborne, A. Amann, K. Buckley, G. Ryan, S. G. Hegarty, G. Huyet, and S. O'Brien, "Antiphase dynamics in a multimode semiconductor laser with optical injection," Phys. Rev. A 79, 023834 (2009).

22. M. Sciamanna and K. Panajotov, "Route to polarization switching induced by optical injection in vertical-cavity surfaceemitting lasers," Phys. Rev. A 73, 023811 (2006).

23. B. Kelleher, C. Bonatto, G. Huyet, and S. P. Hegarty, "Excitability in optically injected semiconductor lasers: contrasting quantum-well and quantum-dot-based devices," Phys. Rev. E 83, 026207 (2011).

24. L. Olejniczak, K. Panajotov, H. Thienpont, and M. Sciamanna, "Self-pulsations and excitability in optically injected quantum dot lasers: impact of the excited states and spontaneous emission noise," Phys. Rev. A 82, 023807 (2010).

25. J. M. Martín-Regalado, F. Prati, M. San Miguel, and N. B. Abraham, "Polarization properties of vertical cavity surface-emitting lasers," IEEE J. Quantum Electron. 33, 765-783 (1997).

26. J. Mulet, C. R. Mirasso, and M. San Miguel, "Polarization resolved intensity noise in vertical-cavity surface-emitting lasers," Phys. Rev. A 64, 023817 (2001).

27. S. C. Chan, S. K. Hwang, and J. M. Liu, "Radio-over fiber AMto-FM upconversion using and optically injected semiconductor laser," Opt. Lett. 31, 2254-2256 (2006). 